Editorial: Hospital Metropolitano

ISSN (impreso) 1390-2989 - ISSN (electrónico) 2737-6303

Edición: Vol. 28 No 3 (2020) Julio - Septiembre

DOI: https://doi.org/10.47464/MetroCiencia/vol28/3/2020/32-38

URL: https://revistametrociencia.com.ec/index.php/revista/article/view/84

Pág: 32-38

\title{
Frecuencia de subtipos moleculares de cáncer de mama, Hospital Metropolitano, 2016-2019, Quito- Ecuador
}

\author{
Frequency of molecular subtypes of breast cancer, \\ Hospital Metropolitano, 2016-2019, Quito- Ecuador
}

\author{
Daniela Vela Merino' ${ }^{1 D}$; Stephania Salazar Chiriboga ${ }^{(i)}$; Danilo Salazar Molina ${ }^{2}$ \\ Médica Cirujana. Posgradista de Ginecología y Obstetricia, Pontificia Universidad Católica del Ecuador ${ }^{1}$ \\ Médico Ginecólogo Obstetra, Hospital Metropolitano, Quito, Ecuador ${ }^{2}$ \\ Médico Rural Centro de Salud Espiga de Oro, Ecuador ${ }^{3}$
}

Recibido: 19/07/2020Ａceptado: 18/08/2020Ｐúblicado: 01/09/2020

\begin{abstract}
RESUMEN
Objetivo: Determinar el tipo molecular más común de CM en el Hospital Metropolitano. Metodología: Se realizó un estudio descriptivo, retrospectivo, en el cual se revisaron los informes histopatológicos de todos los casos de cáncer de mama diagnosticados desde el 01 de enero del 2016 al 31 de diciembre del 2019, teniendo en cuenta los datos inmunohistoquímicos para su clasificación. En el periodo estudiado se evidenció un total de 276 casos correspondientes a cáncer de mama, posterior a la aplicación de los criterios de inclusión se analizaron los datos de 147 pacientes. Resultados: La media anual de casos nuevos fue de $40 \pm 6$. La media de edad al diagnóstico fue de $60.9 \pm 13$ años. La mayoría de pacientes están en el rango de edad de 40 a 69 años con 101 casos (69\%). La mayoría de casos fue de tipo Luminal B con un total de 85 casos, lo cual corresponde al 54\%, la minoría fueron de tipo triple negativo con 11 casos $(7 \%)$. La mayor parte de casos (71\%) tienen elevada profileración tumoral determinada por el valor de Ki-67. Conclusiones: El subtipo molecular de cáncer de mama más común es el Luminal B, y el menos frecuente es el triple negativo. La mayoría de casos tienen alta proliferación determinada por el valor de Ki-67.
\end{abstract}

Palabras claves: Cáncer de mama, Subtipos moleculares, Inmunohistoquímica.

\section{ABSTRACT}

Objective: To determine the most common molecular type of breast cancer in the Hospital Metropolitano. Methodology: This was a retrospective descriptive study, in which the histopathological reports of all breast cancer cases diagnosed from January 1, 2016 to December 31,2019 were reviewed, taking into account immunohistochemical data for classification. In the period studied, a total of 276 cases corresponding to breast cancer were evidenced, after classifying individual criterias, data from 147 patients were analyzed. Results: The annual average of new cases was $40 \pm 6$. The average age at diagnosis was $60.9 \pm 13$ years. A large number of patients are in the age range of 40 to 69 years with 101 cases (69\%). More than a half were Luminal B type with a tota of 85 cases which corresponds to $54 \%$, the minority were triple negative type with 11 cases (7\%). The predominant number of cases ( $71 \%$ ) have high tumor profiling determined by Ki-67. Conclusions: In this retrospective study we found that the most common molecular subtype of breast cancer is Luminal B, and the least frequent is triple negative. The results also demonstrate that most of the cases have high proliferation determined the value of Ki-67.

Keywords: Breast cancer, Molecular subtypes, Immunohistochemistry.

Daniela Vela Merino: Stephania Salazar Chiriboga: Salazar-Molina Danilo:

\section{IDs Orcid}

https://orcid.org/0000-0001-8215-8706 https://orcid.org/0000-0002-6933-0206 https://orcid.org/0000-0003-1730-8186

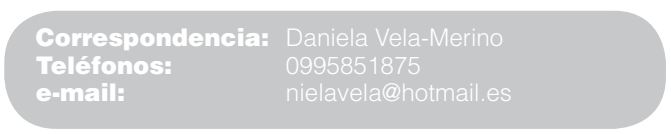




\section{INTRODUCCIÓN}

El cáncer de mama es una proliferación maligna de las células epiteliales que revisten los conductos o lobulillos de la mama. Corresponde a un grupo heterogéneo de enfermedades caracterizado por distintos perfiles moleculares ${ }^{1}$.

Es el tumor maligno más frecuente en el sexo femenino y la principal causa de muerte por cáncer en mujeres de países desarrollados. Según datos del 2018 del Global Cancer Observatory (GLOBOCAN), a nivel mundial se reportaron 2088849 casos, Ios cuales corresponden al 11,66\% entre todos los tipos de cáncer; la tasa de mortalidad es 6,6 de casos por 100000 habitantes. En Ecuador la incidencia es de 31,8 casos por 100000 habitantes con una mortalidad de 9,1 por 100000 habitantes $^{2}$. Se registraron 2787 nuevos casos de CM en el 2018, lo que corresponde a una tasa de 33 por 100000 habitantes, hubo 631 muertes por esta causa, con una tasa de 7.7 por 100000 habitantes $^{2}$.Su incidencia se ha incrementado en los últimos 10 años, a pesar de esto, las tasas de mortalidad anual han disminuido en la última década; esto se atribuye a programas de detección precoz y a los avances en el tratamiento. El cribado tiene el beneficio potencial de evitar el cáncer avanzado y la muerte por $\mathrm{CM}^{3}$.

Acorde a datos del Instituto Nacional de Estadísticas y Censos (INEC), en Ecuador el cáncer de mama es una de las principales causas de muerte en las mujeres, ya que ocupa el lugar número once de la lista de causas generales de muerte femenina del 2017 , en el mismo año se registraron 670 defunciones por cáncer de seno en mujeres y 3 en hombres, lo cual corresponde a una mortalidad de 3.99 defunciones por cada 100000 habitantes $^{4}$.

Existe una relación genética importante entre la herencia y el CM, sin embargo, solo del 3 al 10\% de casos son heredados. El riesgo familiar aumenta según el número de parientes afectados, edad al diagnóstico, tumores ipsilaterales múltiples o bilaterales y antecedente de CM en el sexo masculino². La mayoría de casos son esporádicos y están relacionados con factores potencialmente modificables. El factor de riesgo más importante para desarrollar cáncer de mama es la edad, de esta manera se ha estudiado que el riesgo de desarrollar CM en los siguientes 10 años según edad es ${ }^{6}$ :

- 20 años: 1 en 1732.

- 40 años: 1 en 69.

- 70 años: 1 en 26 .

En situaciones normales la mitosis está regulada por proto-oncogenes y genes supresores de tumores (TSGs). Los proto-oncogenes son los encargados de regular la proliferación celular, mientras que los TSGs regulan el ciclo celular en puntos de control, progresión del ciclo celular, reparación de Ácido Desoxirribonucléico (ADN) dañado y promueven apoptosis, como ejemplo de este tipo de genes tenemos al Breast Cancer Type 1 Suceptibility Protein (BRCA1) y Breast Cancer Type 2 Suceptibility Protein (BRCA2). En oncogénesis el evento inicial es el daño del ADN en un lugar crítico que regula el ciclo celular, lo cual permite proliferación celular no controlada. Posterior a este evento inicial las células tumorales son expuestas a estímulos promotores de tumores y acumulación de daño genético, lo que induce a una proliferación clonal. El estroma asociado al tumor es útil como marco de apoyo, sobre el cual las células tumorales pueden evadir la respuesta inmune, desarrollar neovascularización y capacidad metastásica ${ }^{1}$.

Hanahan \& Weinberg en el 2011 resumen las características de las células cancerígenas en seis puntos $^{7}$ :

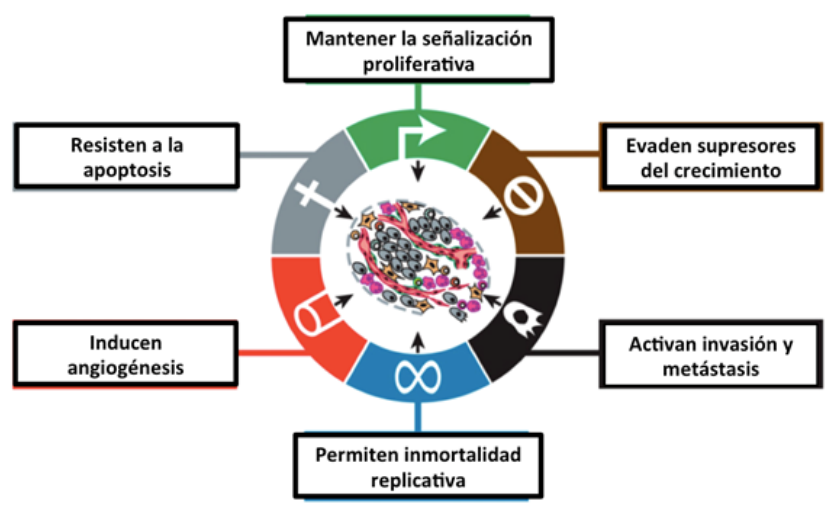

Figura 1. Características de las células cancerígenas.

Modificado de: Hanahan D, Weinberg RA. Hallmarks of cancer: The next generation. Vol. 144, Cell. 2011. p. 646-74.

La clasificación tradicional utilizaba la morfología para dividir los tumores en categorías de acuerdo a características como edad, afectación ganglionar, tamaño y grado tumoral ${ }^{5}$, este sistema tiene limitaciones por lo que en 1970 se comenzó a desarrollar una nueva taxonomía que incluye marcadores específicos, a partir de la cual se puede individualizar el diagnóstico, tratamiento y pronóstico de mejor manera; así se integra el análisis molecular de Receptores de Estrógenos (RE), Receptores de Progesterona $(\mathrm{RP})$, proliferación tumoral determinada por el nivel de la proteína Ki-67, Receptor 2 de Factor de Crecimiento Epidérmico Humano (HER-2), entre otros. Desde el 2004, se dispone de ensayos multigénicos que evalúan aproximadamente 100 genes; en el 2010 se crean paneles genéticos de nueva ge- 
neración que incluyen varios cientos de genes y se determina la secuencia genómica entera con aproximadamente $3 \times 109$ pares de bases ${ }^{1,8}$.

Existen múltiples formas para determinar expresión génica, la utilización de microarreglos y ADN complementario (cADN) es el método de mayor difusión; se basa en la hibridación de moléculas de cADN, preparadas a partir de RNA aislado del tejido tumoral, a secuencias del genoma humano impresas en un soporte sólido.

En la actualidad, se considera que la mejor manera de clasificar al CM es por medio de análisis de los perfiles de expresión génica mediante estudio molecular, hay varias marcas disponibles como Oncotype DX, MammaPrint, Prosigna, EndoPredict, Breast Cancer Index ${ }^{8}$. Sin embargo, su uso es limitado debido a los altos costos y dificultades técnicas.

La inmunohistoquímica (IHQ) es una técnica mediante la cual se puede determinar la presencia y el nivel de ciertas proteínas celulares mediante anticuerpos marcados que se unen a las proteínas de interés ${ }^{9}$. A pesar de haberse demostrado que la mejor manera de determinar un subtipo molecular son los estudios moleculares, existe evidencia de que, de manera rutinaria, los marcadores inmunohistoquímicos bien realizados pueden proporcionar tanta información para ayudar a la toma de decisiones terapéuticas como los estudios moleculares ${ }^{10}$. Es así que en un estudio presentado en el Simposio sobre el cáncer de mama de San Antonio 2009 compararon el poder pronóstico de la puntuación de recurrencia de Oncotype DX, con una fórmula que utiliza cuatro marcadores inmunohistoquímicos estándar ('IHC4'): RE, RP, Ki-67 y HER2 en 1125 mujeres en el ensayo TransATAC. La puntuación de IHC4 mostró una correlación razonable con la puntuación de recurrencia (coeficiente de Pearson 0,7) y proporcionó una cantidad similar de información pronóstico como la puntuación de recurrencia ${ }^{11}$. Estos resultados sugieren que los ensayos $\mathrm{IHQ}$ estándar realizados en un laboratorio de alta calidad pueden proporcionar información de pronóstico similar a los métodos moleculares. Por estas razones, en la práctica hospitalaria mundial y local, el subtipo molecular se determina aplicando técnicas de inmunohistoquímica (IHQ) ${ }^{12}$.

Para resultados confiables se requiere una adecuada preparación del tejido, que inicia desde la extracción del tejido para estudiar, su fijación debe ser inmediata. Se fijan en formalina al 10\% durante 6 a 72 horas. Se procede al embebido en parafina ${ }^{13}$. Posterior a esto se procede a la recuperación antigénica en el tejido mediante calor o inducido por enzimas, ya que la fijación puede causar que el antígeno se enmascare, haciendo imposible que el anticuerpo se una a su objetivo. Se debe evitar falsos positivos causados por peroxidasas endógenas, fosfatasas y biotina, mediante sistemas bloqueadores de estos componentes y de sitios de enlace no específicos a los cuales se pueden unir anticuerpos al tejido erróneamente ${ }^{14}$. La muestra se incuba con el anticuerpo, para posteriormente pasar por diversas fases de lavado, detección del anticuerpo y finalmente un revelado que permite la observación bajo un microscopio de luz'.

El CM se clasifica en subtipos basado en los marcadores inmunohistoquímicos más importantes, de acuerdo a la presencia o ausencia de los siguientes receptores ${ }^{5,15,16}$.

- Receptor de estrógeno: Los cambios fisiológicos mamarios que se dan durante la vida de la mujer, están mediados por el estrógeno. Existen dos tipos de estos receptores:

- RE $\alpha$ : Es un factor pronóstico y predictivo bien establecido en el CM.

- RE $\beta$ : Su importancia no está bien definida, sin embargo, en ocasiones solo se expresa este receptor, lo cual puede conducir a un comportamiento y respuesta clínica diferente. Se ha visto que su expresión disminuye durante la carcinogénesis de mama.

- Receptor de progesterona: De igual manera, hay dos tipos: RP-A y RP-B. La progesterona modula la función estrogénica. En el CM con positividad para RE en ausencia de RP, se ha observado que tienen menor respuesta al tratamiento hormonal que aquellos que expresan RP.

RE y RP no son fenotipos estables ya que pueden cambiar durante la enfermedad o debido al tratamiento.

- HER-2: Un subconjunto de pacientes con CM sobreexpresa el receptor 2 del factor de crecimiento epidérmico humano (HER-2). La sobreexpresión de proteínas generalmente se debe a la amplificación de genes, por lo que los ensayos para determinar el número de copias del gen, la cantidad de ARN mensajero (ARNm) y la proteína generalmente dan resultados similares, de esta manera se estima que la amplificación de genes se correlaciona con la sobreexpresión de proteínas en aproximadamente el 95\% de los casos. La sobreexpresión está implicada en la oncogénesis del CM mediante aumento de la proliferación celular descontrolada, disminución de la apoptosis, aumento de la motilidad de las células cancerosas y angiogénesis, lo cual da como resultado un peor pronóstico.

El estado de HER-2 puede determinarse evaluando la expresión de proteínas en la membrana de las células tumorales usando $\mathrm{IHQ}$ o evaluando el número 
de copias del gen HER-2 con hibridación in situ, los resultados por ambas técnicas deben correlacionarse. En el caso de que se encuentren discrepancias significa que uno de los ensayos es incorrecto, por sobreexpresión de proteínas sin amplificación, amplificación sin sobreexpresión de proteínas o heterogeneidad intratumoral marcada ${ }^{17}$.

- Ki-67: Es una proteína del ciclo celular que se encuentra presente en las células proliferativas, en todas las fases activas del ciclo celular, excepto en la fase G0. El anticuerpo monoclonal MIB-1 es el más utilizado para evaluar Ki-67. El porcentaje de células tumorales positivas para Ki-67 determinado por IHQ se usa a menudo para estratificar a los pacientes en grupos de pronóstico bueno y malo, sin embargo, no hay un consenso universal sobre el punto de corte para definir positividad o negatividad, además hay pocos datos sobre los efectos de las variables preanalíticas (tiempo de isquemia, duración de la fijación, recuperación del antígeno). Por estas razones, ni la American Society of Clinical Oncology (ASCO) ni el National Comprehensive Cancer Network (NCCN) recomiendan la determinación rutinaria de $\mathrm{Ki}-67^{17}$.

Sin embargo, en la práctica se lo utiliza para predecir el riesgo de recurrencia y el potencial benéfico de la quimioterapia en CM con RE+ y ganglios negativos; también se lo considera como factor pronósti$\mathrm{Co}^{17,18}$. Es por esto que se incluye al Ki-67 entre los marcadores inmunohistoquímicos evaluados para determinar el subtipo de CM. El valor de corte para diferenciar a los subtipos Luminal A y Luminal B pueden variar según los estudios. Sin embargo, se ha observado que un nivel inferior a $14 \%$ se relaciona mejor con la expresión genética del Luminal $A$, así como una alta proliferación ( $\geq 14 \%$ ) se correlaciona mejor con Luminal $\mathrm{B}^{19}$, además revela alto contaje mitótico y alto grado tumoral con peor pronóstico que cuando se encuentra en niveles bajos ${ }^{18}$.

Actualmente es indispensable determinar el estado de los receptores mencionados, debido a que se ha demostrado que existe mejor pronóstico en $\mathrm{CM}$ con positividad para receptores de estrógenos y/o progesterona, ya que se pueden beneficiar de un tratamiento con regímenes adyuvantes hormonales o quimioterapéuticos. El estado del RP se asocia independientemente con la supervivencia libre de enfermedad y general ${ }^{20}$.

Basándonos en los tres receptores detallados, el CM se subdivide según su inmunohistoquímica de la siguiente manera ${ }^{21}$ :

- Luminales: Poseen un patrón inmunofenotípico similar al componente epitelial luminal de la glándula mamaria, expresan citoqueratinas lumi- nales, receptores de estrógeno y los genes asociados a su activación (LIV1 y CCND1) ${ }^{22}$.

Existen varios subtipos, los más comunes son $A$ y $B$, de los cuales el más frecuente es el primero correspondiendo al $40 \%$ de los tumores, posee alta expresión de genes relacionados con los receptores hormonales y baja expresión de genes relacionados con la proliferación celular, por lo que se les atribuye el mejor pronóstico; mientras que el subtipo B, es el menos frecuente (20\%), presenta niveles menores de receptores de estrógeno y altos niveles de genes de proliferación ${ }^{22}$.

- HER-2: Su positividad por IHQ se determina por una tinción completa e intensa de la membrana en más del 10\% de las células tumorales. Mientras que por hidridación in situ, se evalúa la presencia o ausencia de amplificación genética ${ }^{17}$. Se caracteriza por mayor riesgo de recaída y mal pronóstico 22 .

Tabla 1. Marcadores inmunohistoquímicos que sustituyen la clasificación molecular.

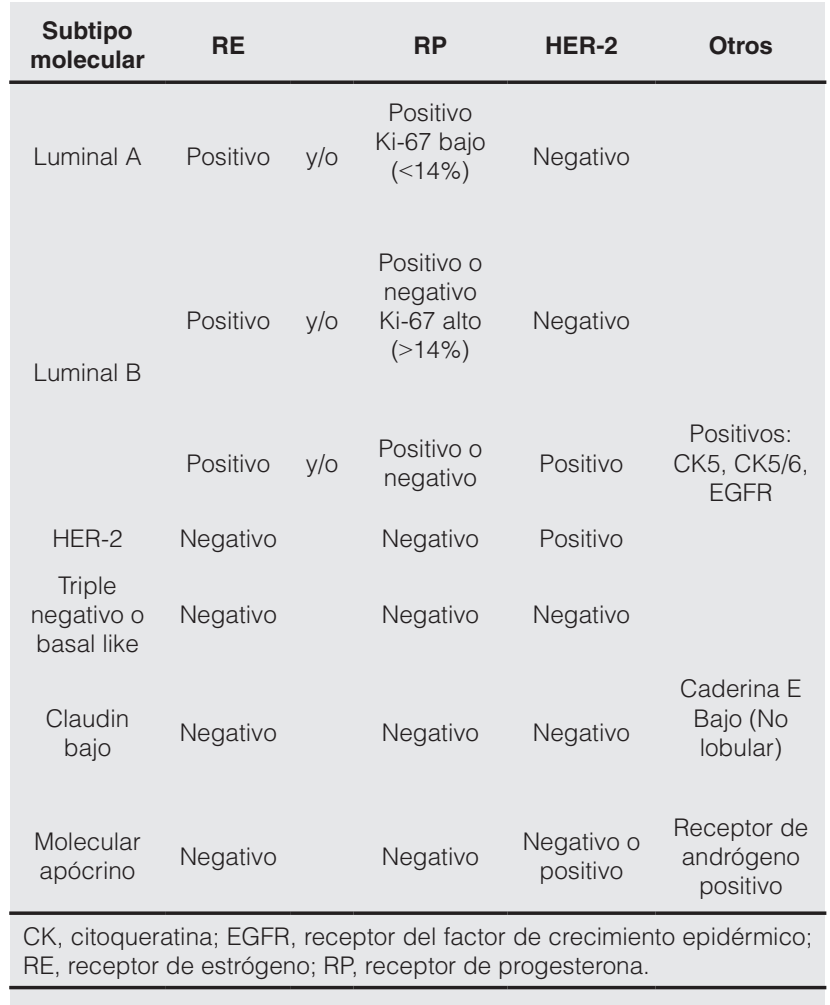

Modificado de Bhargava R. Molecular Classification of Breast Carcinoma. In: Breast Pathology [Internet]. Second Edi. Elsevier Inc.; 2017. p. 417-35. Available from: http://dx.doi.org/10.1016/B978-0323-38961-7.00020-4. 
Actualmente, por $\mathrm{IHQ}$ se reconocen cuatro clases distintas: Iuminal A, luminal B, HER-2 y triple negativo 23 .

El desarrollo de la expresión genética por microarreglos y la tecnología relacionada, proporcionan un perfil más preciso de la enfermedad. La clasificación molecular ofrece más ventajas que la histopatológica como factor predictivo de los diferentes tratamientos, lo cual resulta en un tratamiento más selectivo de la quimioterapia, y con ello la ventaja considerable de reducir toxicidad y $\operatorname{costos}^{24}$. Es así que actualmente los tratamientos combinados con inmunoterapia tienen mejores respuestas, incluso en casos avanzados con metástasis y complicados como los cánceres de subtipo triple negativo, para los cuales se ha desarrollado una subclasificación más específica, en la cual se identifican los puntos de control celular inmunológicos sobre los cuales actúa de manera inhibitoria la inmunoterapia ${ }^{25}$.

\section{MATERIALES Y MÉTODOS}

Se tomó la muestra mediante el sistema GEMA del Hospital Metropolitano, filtrando los CIE10 correspondientes a cáncer de mama (C50), los datos recolectados fueron: edad, diagnóstico, resultados de inmunohistoquímica (RE, RP y Ki-67) e hibridación in situ (HER-2).

En el periodo estudiado se evidenció un total de 276 casos correspondientes a cáncer de mama, de los cuales fueron excluidos los casos con diagnóstico previo, muestra de zonas metastásicas, informes que no contaban con los receptores necesarios para su clasificación, revisión de placas extrahospitalarias. A partir de agosto del 2019 se cambió el método para determinar HER-2, por lo que también se excluyeron estos informes. Se tomaron en cuenta los casos diagnosticados por primera vez, en total se analizaron los datos de 147 pacientes.

Se realizó un estudio descriptivo, retrospectivo, en el cual se revisaron los informes histopatológicos de todos los casos de cáncer de mama diagnosticados desde el 01 de enero 2016 al 31 de diciembre del 2019, teniendo en cuenta los datos inmunohistoquímicos y de hibridación in situ para su clasificación. Se determinó en valor absoluto y relativo la incidencia de cada tipo molecular. Se procesaron los datos mediante el software de ordenador SPSS.

\section{RESULTADOS}

Dentro del periodo estudiado se evidenció que la media anual de casos nuevos es de $40 \pm 6$ (Gráfico $1)$.

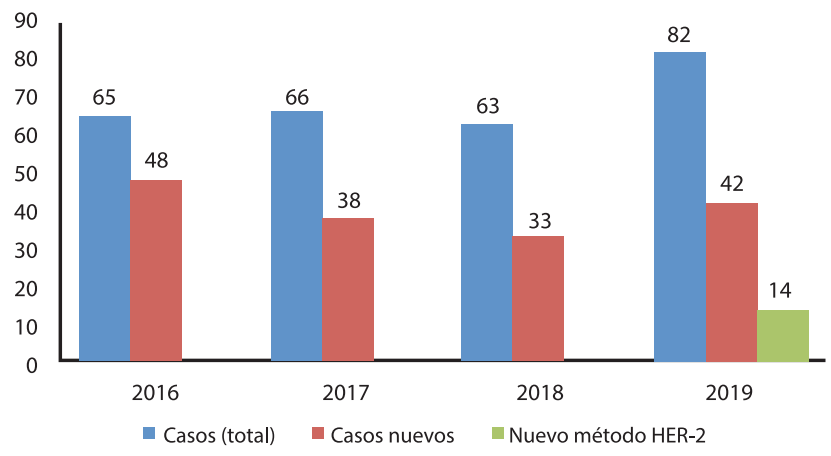

Gráfico 1. Casos de cáncer de mama diagnosticados por año, en azul el total de casos, en rojo los casos nuevos y en verde los casos nuevos tipificados con diferente método.

Fuente: Los autores

La media de edad al diagnóstico fue de 60.9 años con una desviación estándar de 13. Según los grupos etarios estudiados la distribución fue: en el rango de 20 a 39 años hubo un total de 6 pacientes, la mayoría de pacientes están en el rango de edad de 40 a 69 años con 101 casos, en el grupo de $\geq 70$ años el resultado fue de 40 casos. (Gráfico 2).

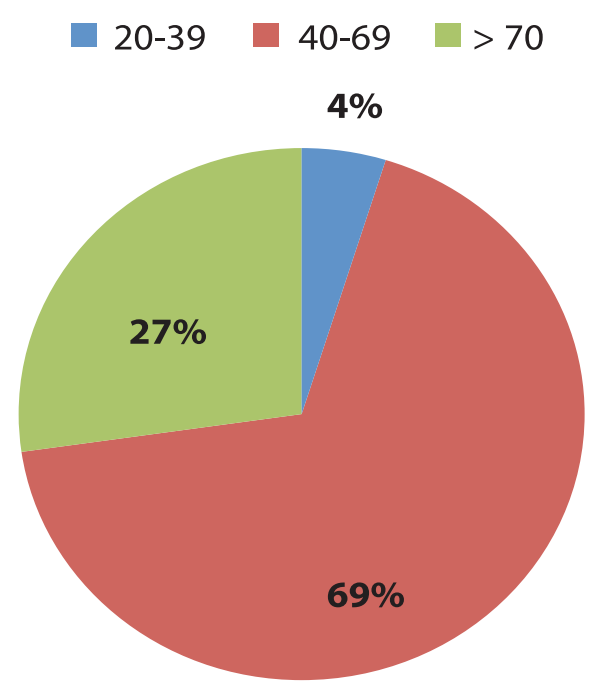

Gráfico 2. Distribución relativa por grupos etarios. En azul de 20 a 39 años, en rojo de 40 a 69 años, en verde $\geq 70$ años.

\section{Fuente: Los autores}

De acuerdo a la clasificación por inmunohistoquímica e hibridación in situ, en el periodo estudiado la mayoría de casos son de tipo Luminal B, con un total de 85 casos, seguido por el Luminal A con 36 casos, del tipo HER-2 15 casos y del tipo triple negativo se evidenciaron 11 casos (Gráfico 3). La distribución anual de los subtipos es similar a la total (Gráfico 4). 
Luminal A - Luminal B $\quad$ HER-2 $\quad$ Triple negativo

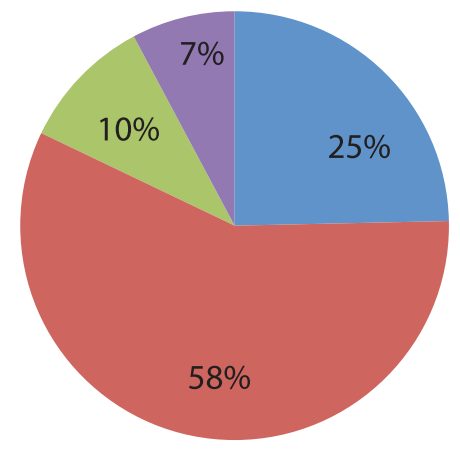

Gráfico 3. Distribución total de cáncer de mama en números relativos según el subtipo molecular determinado por inmunohistoquímica e hibridacio in situ. En azul los Luminal A, rojo Luminal B, verde HER-2, morado triple negativo.

Fuente: Los autores

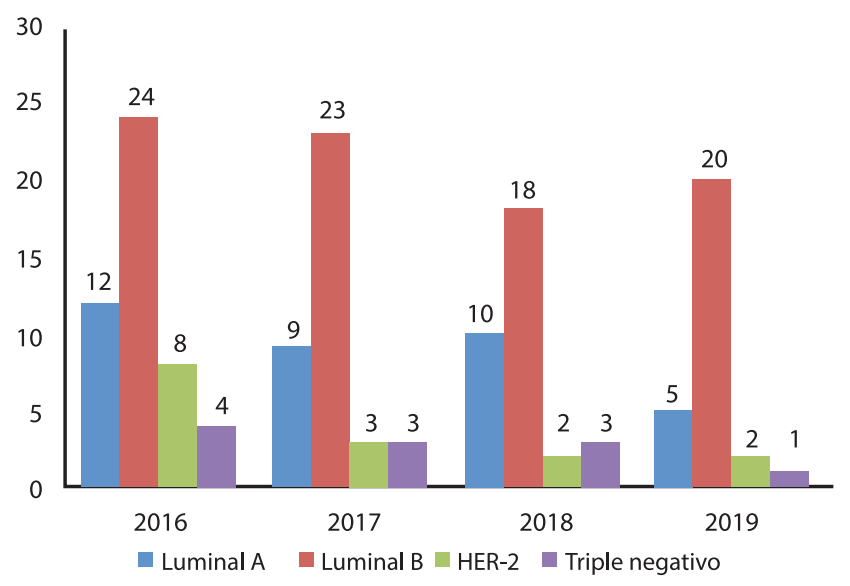

Gráfico 4. Distribución anual de casos de cáncer de mama en números absolutos según el subtipo molecular determinado por inmunohistoquímica e hibridación in situ. En azul los Luminal A, rojo Luminal $B$, verde HER-2, morado triple negativo.

Fuente: Los autores

En cuanto a la profileración tumoral determinada mediante Ki-67 (punto de corte 14\%), se evidencia que la mayoría de casos tienen proliferación tumoral alta con un $71 \%$ (Gráfico 5).
$<14 \%$ (baja) $\quad>14 \%$ (alta)

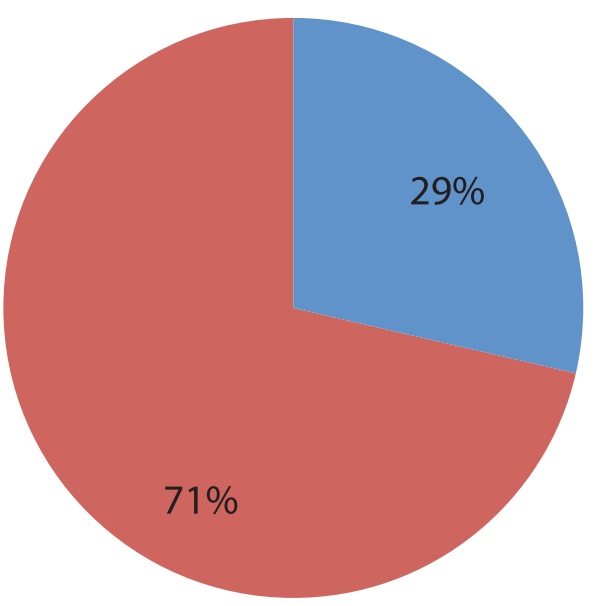

Gráfico 5. Distribución de casos de cáncer de mama en números relativos según la proliferación tumoral determinada por el Ki-67. En azul <14\% (baja), rojo $\geq 14 \%$ (alta).

Fuente: Los autores

\section{CONCLUSIONES}

- Cada año la media de casos nuevos de cáncer de mama diagnosticados en el Hospital Metropolitano es de $40 \pm 6$.

- La mayoría de casos se presentaron en pacientes de entre 40 a 69 años de edad.

- El subtipo molecular de cáncer de mama más común en la población estudiada fue el Luminal $\mathrm{B}, \mathrm{y}$ el menos frecuente fue el triple negativo.

- La mayoría de casos de cáncer de mama diagnosticados en el Hospital Metropolitano tienen alta proliferación, determinada por un índice de proliferación con Ki-67 elevado.

\section{REFERENCIAS BIBLIOGRÁFICAS}

1. Wyld L, Markopoulos C. Breast Cancer Management for Surgeons. Breast Cancer Management for Surgeons. Cham: Springer International Publishing AG; 2018.

2. GLOBOCAN-WHO. Cancer Today [Internet]. International Agency for research. 2018 [cited 2019 Nov 12]. Available from: https://gco.iarc. $\mathrm{fr} /$ today/online-analysis-dual-bars-2?v=2018\&mode=cancer\&mode population $=$ regions $\&$ population $=250 \&$ populations $=250 \&$ key $=$ asr $\&$ sex $=0 \&$ cancer $=39 \&$ type $=0 \&$ statistic $=5 \&$ prevalence $=0$ \& population group=0\&ages_group $\% 5 \mathrm{~B} \% 5 \mathrm{D}=0$ \&ages_group $\% 5 \mathrm{~B} \% 5 \mathrm{D}=17 \& \mathrm{nb}$ items $=10 \& \mathrm{~g}$

3. Viana Zulaica C. Cáncer de mama [Internet]. FISTERRA. 2018. Available from: https://www.clinicalkey.es/\#!/content/guides_techniques/52-s2.0-mt_fis_49

4. Ministerio de Salud Pública del Ecuador. Cifras de Ecuador : Cáncer de Mama [Internet]. Cifras De Ecuador. 2018 [cited 2019 Sep 13]. Available from: https://www.salud.gob.ec/cifras-de-ecuador-cancerde-mamal

5. Kumar N, Patni P, Agarwal A, Khan MA, Parashar N. Prevalence of 
molecular subtypes of invasive breast cancer: A retrospective study. Med J Armed Forces India. 2015;71(3):254-8.

6. Breastcancer.org. Risk of Developing Breast Cancer [Internet]. 2016 [cited 2019 Nov 21]. Available from: https://www.breastcancer.org/ symptoms/understand_bc/risk/understanding

7. Hanahan D, Weinberg RA. Hallmarks of cancer: The next generation. Vol. 144, Cell. 2011. p. 646-74

8. Eliyatkin N, Yalcin E, Zengel B, Aktas S, Vardar E. Molecular Classification of Breast Carcinoma: From Traditional, Old-Fashioned Way to A New Age, and A New Way. J Breast Heal. 2015 Apr 7;11(2):59-66.

9. Emory University. Inmunohistoquímica (IHC) | CancerQuest [Internet]. [cited 2019 Nov 16]. Available from: https://www.cancerquest. org/es/para-los-pacientes/deteccion-y-diagnosis/inmunohistoquimica

10. O'toole SA, Selinger CI, Millar EKA, Lum T, Beith JM. Molecular assays in breast cancerpathology. Royal College of pathologists of Australasia. 2011;43(2):116-27.

11. Cuzick J, Dowsett M, Wale C, Salter J, Quinn E, Zabaglo L, et al. Prognostic Value of a Combined ER, PgR, Ki67, HER2 Immunohistochemical (IHC4) Score and Comparison with the GHI Recurrence Score - Results from TransATAC. Cancer Res [Internet]. 2009 Dec 15 [cited 2020 Aug 20];69(24 Supplement):74-74. Available from: https:// sci-hub.st/https://cancerres.aacrjournals.org/content/69/24_Supplement/74

12. Navarro JM, Castro CS. Inmunohistoquímica en el cáncer de mama. Herramienta necesaria en la actualidad. Medisur [Internet]. 2018 [cited 2019 Nov 21];209-13. Available from: http://scielo.sld.cu/scielo. php?script=sci_arttext\&pid=S1727-897X2018000100016

13. Allison KH, Hammond MEH, Dowsett M, McKernin SE, Carey LA, Fitzgibbons PL, et al. Estrogen and progesterone receptor testing in breast cancer: American society of clinical oncology/college of American pathologists guideline update. Arch Pathol Lab Med [Internet]. 2020 May 1 [cited 2020 Aug 20];144(5):545-63. Available from: http://meridian.allenpress.com/aplm/article/144/5/545/427509/Estrogen-and-Progesterone-Receptor-Testing-in
14. Enzo Life Sciences. Immunohistochemistry E-Book. 2018

15. Almarzooq R, Alrayes A, Alaradi H, Abdulla H. Molecular subtypes of breast cancer. Bahrain Med Bull [Internet]. 2018 [cited 2019 Sep 14];40(4):222-5. Available from: https://www.breastcancer.org/symptoms/types/molecular-subtypes

16. MacKay A, Weigelt B, Grigoriadis A, Kreike B, Natrajan R, A'Hern $\mathbf{R}$, et al. Microarray-based class discovery for molecular classification of breast cancer: Analysis of interobserver agreement. J Natl Cancer Inst. 2011 Apr 20;103(8):662-73.

17. Fitzgibbons PL, Bartley AN, Connolly JL. Template for Reporting Results of Biomarker Testing of Specimens From Patients With Carcinoma of the Breast. Coll Am Pathol. 2020;4(February):1-15.

18. Soliman NA, Yussif SM. Ki-67 as a prognostic marker according to breast cancer molecular subtype. Cancer Biol Med. 2016;13(4):496504.

19. Cáceres V. Tratamiento sistémico: Adyuvancia, parámetros de evaluación para su indicación y utilidad de las firmas genéticas. In: Diagnóstico y tratamiento del cáncer de mama Guía de procedimientos. 1ra ed. Ciudad Autónoma de Buenos Aires; 2016. p. 87-98.

20. Chand P, Anubha G, Singla V, Rani N. Evaluation of immunohistochemical profile of breast cancer for prognostics and therapeutic use. Niger J Surg. 2018;24(2):100.

21. Zaha DC. Significance of immunohistochemistry in breast cancer. Vol. 5, World Journal of Clinical Oncology. Baishideng Publishing Group Co., Limited; 2014. p. 382-92.

22. Witten MO, Krontiras H, Bland FACS KI, Lancaster RB. Molecular Targets in Breast Cancer. In: Current Surgical Therapy. 2020. p. 670-675

23. Bhargava R. Molecular Classification of Breast Carcinoma. In: Breast Pathology. Second Edi. Elsevier Inc.; 2017. p. 417-35.

24. Cleator S, Ashworth A. Molecular profiling of breast cancer: Clinical implications. Vol. 90, British Journal of Cancer. 2004. p. 1120-4.

25. Schmid P. Immune Checkpoint Inhibitors in Triple-Negative Breast Cancer. What Should You Know? In London: Medscape Education; 2019. Available from: https://www.medscape.org/viewarticle/920637_2 